\title{
MVL Toolkit: Software Library for Constructing an Immersive Shared Virtual World
}

\author{
Tetsuro Ogi ${ }^{1,2}$, Takuro Kayahara ${ }^{2}$, Toshio Yamada ${ }^{3}$, Michitaka Hirose $^{2}$ \\ ${ }^{1}$ University of Tsukuba \\ ${ }^{2}$ The University of Tokyo \\ ${ }^{3}$ Gifu Prefectural Research Institute of Manufacturing Infomation Technology \\ tetsu@iml.u-tokyo.ac.jp
}

\begin{abstract}
In this study, we investigated various functions that are required in an immersive shared virtual world, and then developed the MVL Toolkit to implement these functions. The MVL Toolkit contains several utilities that enable such functions as sharing space, sharing users, sharing operations, sharing information and sharing time. By using the MVL Toolkit, collaborative virtual reality applications can be easily constructed by extending existing stand-alone application programs.
\end{abstract}

\section{Introduction}

Recently, virtual reality technology has not only been used for stand-alone applications, but also for networked applications that enable users in remote locations to experience the shared virtual world. However, the software structures of the current collaborative virtual reality applications are often complicated by implementing various functions, such as the collaborative manipulation of objects or the synchronization between remote places. In this study, we investigated the functions that are required in the immersive shared virtual world in order to develop a software library which we called the MVL Toolkit. This library can be used to extend a stand-alone program to a collaborative application without any special redesign of the software by adding several functions.

\section{Elemental Functions}

The required functions in the immersive shared virtual world can be categorized into some elements, such as a) sharing space, b) sharing users, c) sharing operations, d) sharing information and e) sharing time.

In order to construct an immersive shared virtual world, the first requirement is that the same geometric space is shared between both sites. Another requirement is that the user's image is visualized realistically in the threedimensional virtual space so that remote users can see the other user's figure. Thirdly, in order to guarantee the consistency of the shared virtual world, any user's operation that impinges on local environment should also be shared. Moreover, in order to realize collaborative work in the shared virtual world, it is desirable for remote users to be able to share the required information. Although the virtual world is generally updated according to the simulation time at each site, remote users should be able to experience the same world at the same moment.

\section{MVL Toolkit}

In this study, the software library named MVL Toolkit was developed in order to implement the above-mentioned functions. Figure 1 shows the software structure of the MVL Toolkit. The MVL Toolkit consists of several library function sets, such as the $m l S v w$ library, the $m l A v a$ library, the $m l C e l$ library, the $m l C c b$ library and the mlTim library, which were developed corresponding to each of the functions that we identified. Therefore, the user can selectively use the required functions to develop the appropriate collaborative virtual reality application.

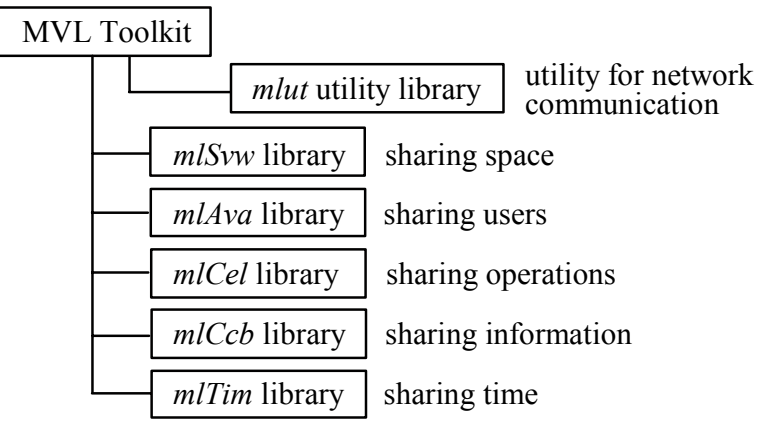

Figure 1. Software structure of MVL Toolkit 
The $m l S v w$ library is used to share the geometric virtual space. In this system, the simplest method of running the same program at both sites is used in order to share the virtual space. These programs must run while communicating with each other and transmitting the data to guarantee the consistency of the shared virtual world.

Next, video avatar technology was used to share the users in the shared virtual world. This method transmits the users' live video images mutually and superimposes them in the virtual world. In particular, the stereo video avatar technique was developed in order to realize high presence communication [1]. This method is implemented using the mlAva library.

In order to share the operations carried out by the user in the virtual world, a cellular phone interface technique was developed. In this method, an interaction server was prepared at one site so that it could receive operation commands that were input by cellular phones from every site and transmit them to the other client sites. These functions are implemented using the $m l C e l$ library.

The $m l C c b$ library realizes the functions of sharing information in the shared virtual world by using the shared-type database system named CCBASE. In this system, the user can access multimedia data through a network, such as images, sound, movie-clips, threedimensional models or PowerPoint files, and then can share these data in the virtual world.

In order to share the correct time in the shared virtual world, the functions governing time passage control and clock adjustment are implemented using the mlTim library. In this method, the time-step is determined according to the elapsed time in every loop, and the clock at the client site is adjusted to the server when the operation data is sent from the server to the clients.

\section{Applications}

In this study, several kinds of collaborative virtual reality applications using the shared virtual world were developed by using the MVL Toolkit in order to evaluate the effectiveness of this library.

Figure 2 shows an application to the flow field visualization system. In this system, analysis data regarding supersonic opposing jet flow were visualized as an animation using several visualization methods, such as streamlining, particles, contours, shading and volume rendering, and remote users discussed with each other while looking at the same visualization data in the immersive shared virtual world. Figure 3 shows another application to the psychological experiment system. In this system, the psychological experiment that investigates how accurately a person's pointing gesture could be recognized by other people was conducted between remote places using the immersive shared virtual world [2].
These systems were developed by extending standalone programs to the collaborative virtual reality applications and the MVL Toolkit was effectively used.

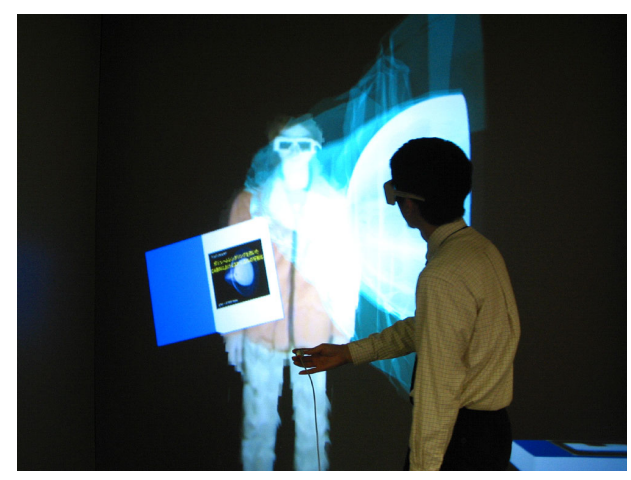

Figure 2. Collaborative flow field visualization

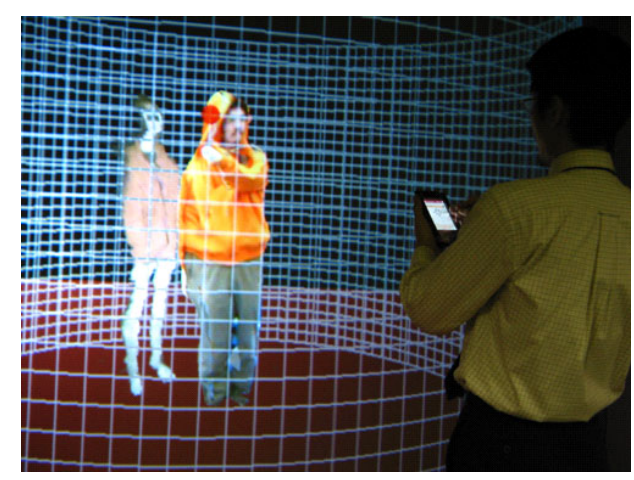

Figure 3. Psychological experiment

\section{Conclusions}

In this study, the functions that are required to construct an immersive shared virtual world were categorized into groups, and they were implemented as the MVL Toolkit. The MVL Toolkit consists of the several library functions, such as sharing space, sharing users, sharing operations, sharing information and sharing time. By using this library, the user can easily develop collaborative virtual reality applications by selectively adding the required functions to a stand-alone program. In this study, the MVL Toolkit was applied to the development of several kinds of applications, and the effectiveness of this library was evaluated.

\section{References}

[1] T. Ogi, T. Yamada, M. Kano, M. Hirose: Immersive Telecommunication Using Stereo Video Avatar, IEEE VR2001, pp.45-51, 2001

[2] T. Ogi, T. Yamada, T. Kayahara: Avatar Communication: Virtual Instructor in the Demonstration Exhibit, HCI International 2003, Vol.3, pp.1431-1435, 2003 